\title{
Entrained Collective Rhythms of Multicellular Systems: Partial Impulsive Control Strategy
}

\author{
Lifei Chen, ${ }^{1}$ Yonghui Sun, ${ }^{2}$ and Qingli Yang ${ }^{2}$ \\ ${ }^{1}$ School of Economics and Management, Nanjing University of Information Science \& Technology, Nanjing 210044, China \\ ${ }^{2}$ College of Energy and Electrical Engineering, Hohai University, Nanjing 210098, China \\ Correspondence should be addressed to Lifei Chen; chenlifei2188@126.com
}

Received 26 September 2013; Accepted 13 November 2013

Academic Editor: Jinde Cao

Copyright (c) 2013 Lifei Chen et al. This is an open access article distributed under the Creative Commons Attribution License, which permits unrestricted use, distribution, and reproduction in any medium, provided the original work is properly cited.

\begin{abstract}
This paper is concerned with the study of entrained collective rhythms of multicellular systems by using partial impulsive control strategy. The objective is to design an impulsive controller based on only those partially available cell states, so that the entrained collective rhythms are guaranteed for the multicellular systems with cell-to-cell communication mechanism. By using the newly developed impulsive integrodifferential inequality, the sufficient conditions are derived to achieve the entrained collective rhythms of multicellular systems. A synthetic multicellular system with simulation results is finally given to illustrate the usefulness of the developed results.
\end{abstract}

\section{Introduction}

Complex physiological rhythms are ubiquitous in living organisms, which are central to life, such as our daily cycle of waking and sleeping and the beating of our hearts. Collective rhythms are normally generated by thousands of divers clock cells which manage to function in a coherent oscillatory state $[1,2]$. In fields ranging from circadian biology to endocrinology, however, it remains an exciting challenge to understand how collective rhythms emerge in multicellular structures [3-7].

Elucidating the collective dynamics of multicellular systems not only is essential for the understanding of the rhythmic phenomena of living organisms at both molecular and cellular levels but also has many potential applications in bioengineering areas. For example, in cancer chemotherapy, treatments could be based on the circadian rhythm of cell division [8]. Over the past decade or so, many researchers have paid a great deal of attention to study the collective dynamics of multicellular systems. For instance, in [9-12], the authors considered stability of genetic networks and neural networks. In [3], the authors pointed out that intercell signaling mechanism does lead to synchronous behavior across a population of cells. In [13], after making real-time analysis of the gene expression, the authors showed the synchronized rhythms of clock gene transcription across hundreds of neurons within the mammalian suprachiasmatic nucleus (SCN) in organotypic slice culture. In addition, based on the Lyapunov stability theory, the collective rhythms of multicellular systems were further studied in [14]. For the other relevant results, please see [15-17].

Although there are significant advances on elucidating the collective behaviors of biological organisms in recent years, the essential mechanisms from which the collective rhythms arise remain to be fully understood. It is well known that coupling among cells is not sufficient to achieve collective rhythms. In fact, the collective rhythms of multicellular systems are far away from being well understood and warrant further and insightful study.

On the other hand, experimental results have already shown that external stimuli play an important role in achieving the collective rhythms. In [18], physiological rhythms were induced by regular or periodic inputs occurring in the context of medical devices. In [19], an external voltage 
was applied to enhance the synchronization of electronic synthetic genetic networks. In [20], it was shown that a specific collective behavior could be realized by changing the frequency and amplitude of the periodic stimuli. Another well-known example is that organisms usually display a circadian rhythm, where the key processes show a 24hour periodicity entrained to the light-dark cycle [21]. In [22], the authors studied the rhythmic process of the circadian oscillators under the effect of the daily light-dark cycle. Furthermore, from the view of impulsive control systems, collective behaviors of coupled systems were investigated and some interesting results have been obtained in [23-27], and, for the other relevant results, please refer to [28-32] and the references therein.

However, in the above-mentioned results, one basic assumption is that the external stimuli are applied to all the cells in the community, that is very expensive or unrealistic in practice. Actually, in many practical medical cases, only partial specific cells could be detected and utilized. In these situations, the external stimuli are applied to only those cells in the community. To the best of our knowledge, there are few results in the open literature on the entrained collective rhythms of multicellular systems by applying impulsive control based on the partially available cell states.

This paper is to study the entrained collective rhythms of multicellular systems with only partially available cell states. By using the newly developed impulsive integrodifferential inequality, a new criterion is derived to ensure the entrained collective rhythms of multicellular systems. It is shown that when the spontaneous synchrony cannot be achieved, an appropriate periodic stimulus could achieve a collective rhythm even only with partially available cell states. It is noted that the proposed partial impulsive control method can be also easily extended to study other complex systems.

The rest of the paper is organized as follows. Section 2 formulates the problem of the entrained collective rhythms and provides some useful lemmas. Section 3 presents the main results for entrained collective rhythms of multicellular systems. A synthetic multicellular system will be employed to illustrate the effectiveness of the developed results in Section 4, which is followed by conclusions in Section 5.

\section{Model Description and Problem Formulation}

To make it easy for the readers, let us start from a single cell model of the form

$$
\dot{x}(t)=-A x(t)+f(x(t)),
$$

where $x(t) \in \mathbb{R}^{n+}$ represents the concentrations of proteins, RNAs, and other chemical complexes, $A$ is the positive diagonal matrix denoting the degradation and dilution rate, and $f(x(t))$ is the complex regulatory function, which usually is of the Michaelis-Menten or Hill form.
Remark 1. It is known that many biological models can be represented by (1), such as the Goodwin model [33] and the toggle switch [34]. Furthermore, the regulatory function $f(x)$ in model (1) is usually monotonically increasing or decreasing.

Without loss of generality, the regulatory function is always assumed to satisfy the following assumption.

Assumption 2. The regulatory function $f(x)$ in (1) satisfies

$$
\left(f_{i}(a)-f_{i}(b)\right)\left[f_{i}(a)-f_{i}(b)-l_{i}(a-b)\right] \leq 0,
$$

for all $a, b \in R, a \neq b$, and $l_{i}>0$.

Consider multicellular systems with cell-to-cell communication mechanism described as follows:

$$
\begin{aligned}
\dot{x}_{i}(t)= & -A x_{i}(t)+f\left(x_{i}(t)\right) \\
& +c \sum_{j=1}^{N} G_{i j} \Gamma x_{j}(t), \quad i=1, \ldots, N
\end{aligned}
$$

where $x_{i}(t) \in \mathbb{R}^{n+}$ is the state of the $i$ th cell, denoting the concentrations of chemical complexes in this cell, and $N$ is the total cell number of the entire community. The third term in model (3) describes the capability of cells to communicate with each other in order to coordinate the behavior of the entire community. $G=\left(G_{i j}\right)_{N \times N}$ is the coupling structure matrix that represents the communications between different cells, and $\Gamma$ is the inner coupling structure that represents the connections of different chemical complexes in one cell. $G$ satisfies the diffusive coupling condition

$$
\begin{gathered}
G_{i j} \geq 0, \quad \text { for } i \neq j, \\
G_{i i}=-\sum_{j=1, j \neq i}^{N} G_{i j}, \quad i=1,2, \ldots, N .
\end{gathered}
$$

It can be noted that such coupling is biologically plausible in many biological systems, such as the quorum sensing mechanism in bacteria $[2,35]$.

Suppose only $l$ cell states in the community are measurable for the multicellular systems (3). Consider the following linear impulsive controller based on those $l$ measurable cell states:

$$
u_{i}=\sum_{k=1}^{\infty} E_{i k}\left(x_{i}-x\right) \delta\left(t-t_{k}\right), \quad i=1,2, \ldots, l, k \in \mathbb{N}
$$

where $x$ is the state of the isolated cell described in (1), $l$ is the number of the measurable cell states, $E_{i k}$ is the gain matrix, and $\delta\left(t-t_{k}\right)$ is the Dirac impulse function with discontinuity points $t_{1}<t_{2}<\cdots<t_{k}<\cdots, \lim _{k \rightarrow \infty} t_{k}=\infty$. 
Then the impulsive-controlled multicellular systems with partial states can be described by the following impulsive differential equation:

$$
\begin{gathered}
\dot{x}_{i}(t)=-A x_{i}(t)+f\left(x_{i}(t)\right) \\
+c \sum_{j=1}^{N} G_{i j} \Gamma x_{j}(t), \quad t \in\left(t_{k-1}, t_{k}\right], \\
\Delta x_{i}\left(t_{k}\right)=x_{i}\left(t_{k}^{+}\right)-x_{i}\left(t_{k}^{-}\right)=E_{i k}\left(x_{i}-x\right), \\
k=1,2, \ldots, \quad t=t_{k}, \\
\dot{x}_{i}(t)=-A=1, \ldots, l, \\
+c \sum_{i}(t)+f\left(x_{i}(t)\right) \quad i=l+1, \ldots, N .
\end{gathered}
$$

Defining $e_{i}(t)=x_{i}(t)-x(t)$, one can obtain the following error system:

$$
\begin{gathered}
\dot{e}_{i}(t)=-A e_{i}(t)+f\left(x_{i}(t)\right)-f(x(t)) \\
+c \sum_{j=1}^{N} G_{i j} \Gamma e_{j}(t), \quad t \in\left(t_{k-1}, t_{k}\right], \\
e_{i}\left(t_{k}^{+}\right)=\left(I+E_{i k}\right) e_{i}\left(t_{k}\right), \quad k=1,2, \ldots, \\
t=t_{k}, \quad i=1, \ldots, l, \\
\dot{e}_{i}(t)=-A e_{i}(t)+f\left(x_{i}(t)\right)-f(x(t)) \\
+c \sum_{j=1}^{N} G_{i j} \Gamma e_{j}(t), \quad i=l+1, \ldots, N .
\end{gathered}
$$

Then the problem of entrained collective rhythms is to design the partial impulsive controller (5) such that the stability of the error system (7) is guaranteed. Before presenting the main results, some useful lemmas are introduced in advance.

Lemma 3 (see [36]). If $P \in \mathbb{R}^{n \times n}$ is a positive definite matrix and $Q \in \mathbb{R}^{n \times n}$ is a symmetric matrix, then

$$
\begin{aligned}
& \lambda_{\min }\left(P^{-1} Q\right) x^{\mathrm{T}} P x \leq x^{\mathrm{T}} \mathrm{Q} x \\
& \quad \leq \lambda_{\max }\left(P^{-1} Q\right) x^{\mathrm{T}} P x, \quad \forall x \in \mathbb{R}^{n},
\end{aligned}
$$

where $\lambda_{\min }(\cdot)$ and $\lambda_{\max }(\cdot)$ are the minimum and maximum eigenvalues of the matrix, respectively.

Lemma 4. For positive scalars $\delta>0, \mu>0$, and $\rho>$ 0 , if $u(t)$ satisfies

$$
\begin{gathered}
\dot{u}(t) \leq h(t, u(t))+\delta \int_{0}^{t} e^{-\rho(t-s)} u(s) d s, \quad t \neq t_{k}, \\
u\left(t_{k}^{+}\right) \leq \mu u\left(t_{k}\right), \quad t=t_{k}, \\
u(0) \leq u_{0},
\end{gathered}
$$

where $h(t, u(t))$ is a continuous function, then one has $u(t) \leq$ $u_{\epsilon}(t), t \geq 0$ for $\epsilon \geq 0$, where $u_{\epsilon}(t)$ is the solution to the following impulsive integrodifferential equation:

$$
\begin{gathered}
\dot{u}_{\epsilon}(t)=h\left(t, u_{\epsilon}(t)\right)+\delta \int_{0}^{t} e^{-\rho(t-s)} u_{\epsilon}(s) d s+\epsilon, \quad t \neq t_{k}, \\
u_{\epsilon}\left(t_{k}^{+}\right)=\mu u_{\epsilon}\left(t_{k}\right), \quad t=t_{k}, \\
u_{\epsilon}(0)=u_{0} .
\end{gathered}
$$

Proof. Firstly, we prove

$$
u(t) \leq u_{\epsilon}(t), \quad t \in\left(0, t_{1}\right] .
$$

If argument (11) is not right, then there exists $\widetilde{t} \in\left(0, t_{1}\right)$ such that

$$
u(\widetilde{t})>u_{\epsilon}(\tilde{t}) .
$$

Considering the continuity of $u(t), u_{\epsilon}(t)$ on $\left(0, t_{1}\right]$, there must exist $t^{*} \in(0, \widetilde{t})$ such that

$$
\begin{gathered}
u\left(t^{*}\right)=u_{\epsilon}\left(t^{*}\right), \quad \dot{u}\left(t^{*}\right) \geq \dot{u}_{\epsilon}\left(t^{*}\right), \\
u(t) \leq u_{\epsilon}(t), \quad \forall t \leq t^{*}
\end{gathered}
$$

then it yields

$$
\begin{aligned}
\dot{u}\left(t^{*}\right) & \leq h\left(t^{*}, u\left(t^{*}\right)\right)+\delta \int_{0}^{t^{*}} e^{-\rho\left(t^{*}-s\right)} u(s) d s \\
& <h\left(t^{*}, u_{\epsilon}\left(t^{*}\right)\right)+\delta \int_{0}^{t^{*}} e^{-\rho\left(t^{*}-s\right)} u_{\epsilon}(s) d s+\epsilon \\
& =\dot{u}_{\epsilon}\left(t^{*}\right),
\end{aligned}
$$

which contradicts the condition $\dot{u}\left(t^{*}\right) \geq \dot{u}_{\epsilon}\left(t^{*}\right)$, so (11) holds.

Suppose $u(t) \leq u_{\epsilon}(t)$, for all $t \in\left(0, t_{k}\right]$; then by $u\left(t_{k}^{+}\right)=$ $\mu u\left(t_{k}\right) \leq \mu u_{\epsilon}\left(t_{k}\right)=u_{\epsilon}\left(t_{k}^{+}\right)$, similarly, one has $u(t) \leq u_{\epsilon}(t)$, for all $t \in\left(t_{k}, t_{k+1}\right]$. By using the mathematical induction method, one can conclude $u(t) \leq u_{\epsilon}(t)$, for all $t \in\left(0, t_{k}\right]$ for any positive integer $k$. The proof is thus complete.

Lemma 5 (Grownwall-Bellman Inequality [37]). Let $\alpha(t)$ be a real value continuous function and $\beta(t)$ a nonnegative continuous function on $[a, b]$. If a continuous function $u(t)$ has the property that

$$
u(t) \leq \alpha(t)+\int_{a}^{t} \beta(s) u(s) d s, \quad a \leq t \leq b,
$$

then on $[a, b]$ one has

$$
u(t) \leq \alpha(t)+\int_{a}^{t} \alpha(s) \beta(s) \exp \left(\int_{s}^{t} \beta(r) d r\right) d s .
$$

Definition 6. The multicellular system (6) is said to achieve collective rhythms with the designed partial impulsive controller, if there exist scalars $\rho>0$ and $M>0$ such that

$$
\lim _{t \rightarrow \infty}\|X(t)\|^{2} \leq M e^{-\rho t}\|X(0)\|^{2}
$$


where $X(t)=\left[x_{1}^{\mathrm{T}}-x^{\mathrm{T}}, \ldots, x_{N}^{\mathrm{T}}-x^{\mathrm{T}}\right]^{\mathrm{T}}$ and $X(0)$ is the initial condition.

\section{Main Results}

In this section, by using the proposed impulsive integrodifferential inequality, a sufficient condition guaranteeing the entrained collective rhythms of multicellular systems is derived.

Theorem 7. For a given scalar $\mu \in(0,1)$, if there exist matrices $P_{i}>0, i=1,2, \ldots, l, Q_{i}>0, i=l+1, \ldots, N$, scalars $\lambda_{i}>0$, $\mu_{i}>0, i=1,2, \ldots, l, \eta_{i}>0, i=l+1, \ldots, N$, and positive scalars $\epsilon_{1}, \epsilon_{2}, \delta_{1}$, and $\delta_{2}$ such that

$$
\begin{gathered}
\left(-A^{T} P_{i}-P_{i} A\right)+\lambda_{\max }\left(P_{i}\right)\left(\epsilon_{1} I+\epsilon_{1}^{-1} L^{T} L+c \delta_{1} \sum_{j=1}^{N}\left|G_{i j}\right|\right) \\
+c \delta_{1}^{-1} \sum_{j=1}^{l} \lambda_{\max }\left(P_{j}\right)\left|G_{j i}\right| \Gamma^{T} \Gamma \leq \lambda_{i} P_{i},
\end{gathered}
$$$$
\left(I+E_{i k}\right)^{T} P_{i}\left(I+E_{i k}\right) \leq \mu_{i} P_{i},
$$

$$
\begin{aligned}
& \text { for } i=1, \ldots, l \text {, and } \\
& \qquad \begin{array}{l}
\left(-A^{T} Q_{i}-Q_{i} A\right)+\lambda_{\max }\left(Q_{i}\right)\left(\epsilon_{2} I+\epsilon_{2}^{-1} L^{T} L+c \delta_{2} \sum_{j=1}^{N}\left|G_{i j}\right|\right) \\
\quad+c \delta_{2}^{-1} \sum_{j=l+1}^{N} \lambda_{\max }\left(Q_{j}\right)\left|G_{j i}\right| \Gamma^{T} \Gamma \leq-\eta_{i} Q_{i},
\end{array}
\end{aligned}
$$

for $i=l+1, \ldots, N$, and, for any impulsive time sequence $\left\{t_{k}\right\}$ satisfying

$$
\delta:=\sup _{k}\left\{t_{k+1}-t_{k}\right\}<\frac{\ln (1 / \mu)}{\eta+\lambda+(\beta \gamma) /(\eta \mu)},
$$

for $k=0,1,2, \ldots$, where $\mu=\max \left(\mu_{i}\right), \lambda=\max \left(\lambda_{i}\right), i=$ $1, \ldots, l$, and $\eta=\min \left(\eta_{i}\right), i=l+1, \ldots, N$, then the entrained collective rhythms of multicellular systems (6) are achieved.

Proof. Consider the following Lyapunov function:

$$
V(t)=V_{1}(t)+V_{2}(t)
$$

where

$$
\begin{aligned}
V_{1}(t) & :=\sum_{i=1}^{l} e_{i}^{\mathrm{T}}(t) P_{i} e_{i}(t), \\
V_{2}(t) & :=\sum_{i=l+1}^{N} e_{i}^{\mathrm{T}}(t) Q_{i} e_{i}(t),
\end{aligned}
$$

where $P_{i}, i=1,2, \ldots, l$ and $Q_{i}, i=l+1, \ldots, N$ are positive definite matrices to be determined.

For any $t \in\left(t_{k}, t_{k+1}\right], k \in \mathbb{N}$, taking the Dini derivative along the trajectories of (7), we have

$$
\begin{aligned}
D^{+} V_{1}(t)=\sum_{i=1}^{l}\left(\dot{e}_{i}^{\mathrm{T}}(t) P_{i} e_{i}(t)+e_{i}^{\mathrm{T}}(t) P_{i} \dot{e}_{i}(t)\right) \\
=\sum_{i=1}^{l}\left(e_{i}^{\mathrm{T}}(t)\left(-A^{\mathrm{T}} P_{i}-P_{i} A\right) e_{i}(t)\right. \\
+2 e_{i}^{\mathrm{T}}(t) P_{i}\left(f\left(x_{i}(t)\right)-f(x(t))\right) \\
\left.+2 c \sum_{j=1}^{N} e_{i}^{\mathrm{T}}(t) P_{i} G_{i j} \Gamma e_{j}(t)\right) .
\end{aligned}
$$

It follows from Assumption 2 that

$$
\begin{aligned}
& 2 e_{i}^{\mathrm{T}}(t) P_{i}\left(f\left(x_{i}(t)\right)-f(x(t))\right) \\
& \leq \lambda_{\max }\left(P_{i}\right)\left(\epsilon_{1} e_{i}^{\mathrm{T}}(t) e_{i}(t)+\epsilon_{1}^{-1} e_{i}^{\mathrm{T}}(t) L^{\mathrm{T}} L e_{i}(t)\right), \\
& 2 c \sum_{j=1}^{N} e_{i}^{\mathrm{T}}(t) P_{i} G_{i j} \Gamma e_{j}(t) \\
& \leq c \lambda_{\max }\left(P_{i}\right) \sum_{j=1}^{N}\left|G_{i j}\right| \\
& \quad \times\left(\delta_{1} e_{i}^{\mathrm{T}}(t) e_{i}(t)+\delta_{1}^{-1} e_{j}^{\mathrm{T}}(t) \Gamma^{\mathrm{T}} \Gamma e_{j}(t)\right),
\end{aligned}
$$

where $\epsilon_{1}>0, \delta_{1}>0$ and $L=\operatorname{diag}\left(l_{1}, \ldots, l_{n}\right)$. One also has

$$
\begin{gathered}
c \delta_{1}^{-1} \sum_{i=1}^{l} \sum_{j=1}^{N} \lambda_{\max }\left(P_{i}\right)\left|G_{i j}\right| e_{j}^{\mathrm{T}}(t) \Gamma^{\mathrm{T}} \Gamma e_{j}(t) \\
=c \delta_{1}^{-1} \sum_{i=1}^{N} \sum_{j=1}^{l} \lambda_{\max }\left(P_{j}\right)\left|G_{j i}\right| e_{i}^{\mathrm{T}}(t) \Gamma^{\mathrm{T}} \Gamma e_{i}(t) \\
=c \delta_{1}^{-1} \sum_{i=1}^{l} \sum_{j=1}^{l} \lambda_{\max }\left(P_{j}\right)\left|G_{j i}\right| e_{i}^{\mathrm{T}}(t) \Gamma^{\mathrm{T}} \Gamma e_{i}(t) \\
+c \delta_{1}^{-1} \sum_{i=l+1}^{N} \sum_{j=1}^{l} \lambda_{\max }\left(P_{j}\right)\left|G_{j i}\right| e_{i}^{\mathrm{T}}(t) \Gamma^{\mathrm{T}} \Gamma e_{i}(t) .
\end{gathered}
$$


Substituting (25)-(26) into (24) yields

$$
\begin{aligned}
& D^{+} V_{1}(t) \leq \sum_{i=1}^{l}\left(e_{i}^{\mathrm{T}}(t)\left(-A^{\mathrm{T}} P_{i}-P_{i} A\right) e_{i}(t)+\lambda_{\max }\left(P_{i}\right) e_{i}^{\mathrm{T}}(t)\right. \\
& \times\left(\epsilon_{1} I+\epsilon_{1}^{-1} L^{\mathrm{T}} L+c \delta_{1} \sum_{j=1}^{N}\left|G_{i j}\right|\right) e_{i}(t) \\
& \left.+c \delta_{1}^{-1} \sum_{i=1}^{l} \sum_{j=1}^{l} \lambda_{\max }\left(P_{j}\right)\left|G_{j i}\right| e_{i}^{\mathrm{T}}(t) \Gamma^{\mathrm{T}} \Gamma e_{i}(t)\right) \\
& +c \delta_{1}^{-1} \sum_{i=l+1}^{N} \sum_{j=1}^{l} \lambda_{\max }\left(P_{j}\right)\left|G_{j i}\right| e_{i}^{\mathrm{T}}(t) \Gamma^{\mathrm{T}} \Gamma e_{i}(t) \\
& =\sum_{i=1}^{l} e_{i}^{\mathrm{T}}(t)\left(\left(-A^{\mathrm{T}} P_{i}-P_{i} A\right)+\lambda_{\max }\left(P_{i}\right)\right. \\
& \times\left(\epsilon_{1} I+\epsilon_{1}^{-1} L^{\mathrm{T}} L+c \delta_{1} \sum_{j=1}^{N}\left|G_{i j}\right|\right) \\
& \left.+c \delta_{1}^{-1} \sum_{j=1}^{l} \lambda_{\max }\left(P_{j}\right)\left|G_{j i}\right| \Gamma^{\mathrm{T}} \Gamma\right) e_{i}(t) \\
& +c \delta_{1}^{-1} \sum_{i=l+1}^{N} \sum_{j=1}^{l} \lambda_{\max }\left(P_{j}\right)\left|G_{j i}\right| e_{i}^{\mathrm{T}}(t) \Gamma^{\mathrm{T}} \Gamma e_{i}(t) .
\end{aligned}
$$

Define $\beta_{i}=c \delta_{1}^{-1} \sum_{j=1}^{l} \lambda_{\max }\left(P_{j}\right)\left|G_{j i}\right|$. Then it follows from condition (18) and Lemma 3 that

$$
\begin{aligned}
D^{+} V_{1}(t) & \leq \sum_{i=1}^{l} e_{i}^{\mathrm{T}}(t) \lambda_{i} P_{i} e_{i}(t)+\sum_{i=l+1}^{N} \beta_{i} e_{i}^{\mathrm{T}}(t) e_{i}(t) \\
& \leq \lambda V_{1}(t)+\beta V_{2}(t),
\end{aligned}
$$

where $\lambda=\max \left(\lambda_{i}\right)$ and $\beta=\max \left(\beta_{i} \lambda_{\max }\left(Q_{i}^{-1}\right)\right)$.

Furthermore, for any $t \geq 0$, one can also get

$$
\begin{aligned}
D^{+} V_{2}(t)= & \sum_{i=l+1}^{N}\left(\dot{e}_{i}^{\mathrm{T}}(t) Q_{i} e_{i}(t)+e_{i}^{\mathrm{T}}(t) Q_{i} \dot{e}_{i}(t)\right) \\
= & \sum_{i=l+1}^{N}\left(e_{i}^{\mathrm{T}}(t)\left(-A^{\mathrm{T}} Q_{i}-Q_{i} A\right) e_{i}(t)\right. \\
& +2 e_{i}^{\mathrm{T}}(t) Q_{i}\left(f\left(x_{i}(t)\right)-f(x(t))\right) \\
& \left.+2 c \sum_{j=1}^{N} e_{i}^{\mathrm{T}}(t) Q_{i} G_{i j} \Gamma e_{j}(t)\right)
\end{aligned}
$$

$$
\begin{aligned}
& \leq \sum_{i=l+1}^{N}\left(e_{i}^{\mathrm{T}}(t)\left(-A^{\mathrm{T}} Q_{i}-Q_{i} A\right) e_{i}(t)\right. \\
& +\lambda_{\text {max }}\left(Q_{i}\right) e_{i}^{\mathrm{T}}(t) \\
& \times\left(\epsilon_{2} I+\epsilon_{2}^{-1} L^{\mathrm{T}} L+c \delta_{2} \sum_{j=1}^{N}\left|G_{i j}\right|\right) e_{i}(t) \\
& \left.+c \delta_{2}^{-1} \sum_{j=l+1}^{N} \lambda_{\max }\left(Q_{j}\right)\left|G_{j i}\right| e_{i}^{\mathrm{T}}(t) \Gamma^{\mathrm{T}} \Gamma e_{i}(t)\right) \\
& +c \delta_{2}^{-1} \sum_{i=1}^{l} \sum_{j=l+1}^{N} \lambda_{\max }\left(Q_{j}\right)\left|G_{j i}\right| e_{i}^{\mathrm{T}}(t) \Gamma^{\mathrm{T}} \Gamma e_{i}(t) \\
& =\sum_{i=l+1}^{N} e_{i}^{\mathrm{T}}(t) \\
& \times\left(\left(-A^{\mathrm{T}} Q_{i}-Q_{i} A\right)+\lambda_{\max }\left(Q_{i}\right)\right. \\
& \times\left(\epsilon_{2} I+\epsilon_{2}^{-1} L^{\mathrm{T}} L+c \delta_{2} \sum_{j=1}^{N}\left|G_{i j}\right|\right) \\
& \left.+c \delta_{2}^{-1} \sum_{j=l+1}^{N} \lambda_{\max }\left(Q_{j}\right)\left|G_{j i}\right| \Gamma^{\mathrm{T}} \Gamma\right) e_{i}(t) \\
& +c \delta_{2}^{-1} \sum_{i=1}^{l} \sum_{j=l+1}^{N} \lambda_{\max }\left(Q_{j}\right)\left|G_{j i}\right| e_{i}^{\mathrm{T}}(t) \Gamma^{\mathrm{T}} \Gamma e_{i}(t),
\end{aligned}
$$

where $\epsilon_{2}>0, \delta_{2}>0$.

Defining $\gamma_{i}=c \delta_{2}^{-1} \sum_{j=l+1}^{N} \lambda_{\max }\left(Q_{j}\right)\left|G_{j i}\right|$, it follows from (20) and (29) that

$$
\begin{aligned}
D^{+} V_{2}(t) \leq & -\sum_{i=l+1}^{N} \eta_{i} e_{i}^{\mathrm{T}}(t) Q_{i} e_{i}(t) \\
& +\sum_{i=1}^{l} \gamma_{i} e_{i}^{\mathrm{T}}(t) e_{i}(t) \\
\leq & -\eta V_{2}(t)+\gamma V_{1}(t),
\end{aligned}
$$

where $\eta=\min \left(\eta_{i}\right)$ and $\gamma=\max \left(\gamma_{i} \lambda_{\max }\left(P_{i}^{-1}\right)\right)$, which implies

$$
V_{2}(t) \leq e^{-\eta t} V_{2}(0)+\gamma \int_{0}^{t} e^{-\eta(t-\alpha)} V_{1}(\alpha) d \alpha .
$$

Then substituting (31) into (28) yields

$$
\begin{aligned}
D^{+} V_{1}(t) \leq & \lambda V_{1}(t)+\beta e^{-\eta t} V_{2}(0) \\
& +\beta \gamma \int_{0}^{t} e^{-\eta(t-\alpha)} V_{1}(\alpha) d \alpha, \quad t \in\left(t_{k}, t_{k+1}\right] .
\end{aligned}
$$


On the other hand, when $t=t_{k}^{+}$, it follows from (19) that

$$
\begin{aligned}
V_{1}\left(t_{k}^{+}\right) & =\sum_{i=1}^{l} e_{i}^{\mathrm{T}}\left(t_{k}\right)\left(I+E_{i k}\right)^{\mathrm{T}} P_{i}\left(I+E_{i k}\right) e_{i}\left(t_{k}\right) \\
& \leq \sum_{i=1}^{l} e_{i}^{\mathrm{T}}\left(t_{k}\right) \mu_{i} P_{i} e_{i}\left(t_{k}\right) \\
& \leq \mu V_{1}\left(t_{k}\right)
\end{aligned}
$$

where $\mu=\max \left(\mu_{i}\right), i=1, \ldots, l$.

For any scalar $\epsilon>0$, define the following impulsive integrodifferential equation:

$$
\begin{gathered}
\dot{V}_{\epsilon}(t)=\lambda V_{\epsilon}(t)+\beta e^{-\eta t} V_{2}(0) \\
+\beta \gamma \int_{0}^{t} e^{-\eta(t-\alpha)} V_{\epsilon}(\alpha) d \alpha \\
+\epsilon, \quad t \in\left(t_{k-1}, t_{k}\right], \\
V_{\epsilon}\left(t_{k}^{+}\right)=\mu V_{\epsilon}\left(t_{k}\right), \quad t=t_{k}, \\
V_{\epsilon}(0)=V_{1}(0) .
\end{gathered}
$$

It then follows from Lemma 4 that $V_{1}(t) \leq V_{\epsilon}(t)$, for all $t \in$ $\left(0, t_{k}\right]$.

The solution to (34) can be expressed as follows:

$$
\begin{aligned}
V_{\epsilon}(t)= & W(t, 0) V_{\epsilon}(0)+\int_{0}^{t} W(t, s) \\
\times & \left\{\epsilon+\beta e^{-\eta s} V_{2}(0)\right. \\
& \left.+\beta \gamma \int_{0}^{s} e^{-\eta(s-\alpha)} V_{\epsilon}(\alpha) d \alpha\right\} d s,
\end{aligned}
$$

where $W(t, s)(t, s \geq 0)$ is the Cauchy matrix of the linear impulsive system

$$
\begin{gathered}
\dot{u}(t)=\lambda u(t), \quad t \in\left(t_{k-1}, t_{k}\right], \\
u\left(t_{k}^{+}\right)=\mu u\left(t_{k}\right), \quad k=1,2, \ldots, t=t_{k} .
\end{gathered}
$$

Furthermore, noting that $\mu<1$ and $t_{k+1}-t_{k} \leq \delta$, it follows from condition $(21)$ that $W(t, s)$ could be estimated as

$$
\begin{aligned}
W(t, s) & =e^{\lambda(t-s)} \prod_{s<t_{k} \leq t} \mu \leq e^{\lambda(t-s)} \mu^{((t-s) / \delta)-1} \\
& =\frac{1}{\mu} e^{-((1 / \delta) \ln (1 / \mu)-\lambda)(t-s)} .
\end{aligned}
$$

Defining $\widehat{\lambda}=(1 / \delta) \ln (1 / \mu)-\lambda$, one has

$$
\begin{aligned}
V_{\epsilon}(t) \leq & \frac{1}{\mu} e^{-\widehat{\lambda} t} V_{\epsilon}(0) \\
& +\frac{1}{\mu} \int_{0}^{t} e^{-\widehat{\lambda}(t-s)}\left(\epsilon+\beta e^{-\eta s} V_{2}(0)\right) d s \\
& +\frac{\beta \gamma}{\mu} \int_{0}^{t} e^{-\widehat{\lambda}(t-s)} \int_{0}^{s} e^{-\eta(s-\alpha)} V_{\epsilon}(\alpha) d \alpha d s \\
= & \frac{1}{\mu} e^{-\widehat{\lambda} t} V_{\epsilon}(0)+\frac{\epsilon}{\mu \widehat{\lambda}}\left(1-e^{-\hat{\lambda} t}\right) \\
& +\frac{\beta V_{2}(0)}{\mu(\widehat{\lambda}-\eta)}\left(e^{-\eta t}-e^{-\widehat{\lambda} t}\right) \\
& +\frac{\beta \gamma}{\mu} \int_{0}^{t} \int_{\alpha}^{t} e^{-\widehat{\lambda}(t-s)} e^{-\eta(s-\alpha)} V_{\epsilon}(\alpha) d s d \alpha \\
= & \frac{1}{\mu} e^{-\widehat{\lambda} t} V_{\epsilon}(0)+\frac{\epsilon}{\mu \widehat{\lambda}}\left(1-e^{-\widehat{\lambda} t}\right) \\
& +\frac{\beta V_{2}(0)}{\mu(\widehat{\lambda}-\eta)}\left(e^{-\eta t}-e^{-\widehat{\lambda} t}\right)+\frac{\beta \gamma}{\mu(\widehat{\lambda}-\eta)} \\
& \times \int_{0}^{t}\left(e^{-\eta(t-\alpha)}-e^{-\hat{\lambda}(t-\alpha)}\right) V_{\epsilon}(\alpha) d \alpha . \\
&
\end{aligned}
$$

Noting from condition (21) that $\hat{\lambda}>\eta$, then one gets

$$
\begin{aligned}
V_{\epsilon}(t) \leq & \frac{1}{\mu} e^{-\hat{\lambda} t} V_{\epsilon}(0)+\frac{\epsilon}{\mu \widehat{\lambda}}+\frac{\beta V_{2}(0)}{\mu(\widehat{\lambda}-\eta)} e^{-\eta t} \\
& +\frac{\beta \gamma}{\mu(\widehat{\lambda}-\eta)} \int_{0}^{t} e^{-\eta(t-\alpha)} V_{\epsilon}(\alpha) d \alpha .
\end{aligned}
$$

Defining $u(t)=e^{\eta t} V_{\epsilon}(t), \alpha(t)=\left(V_{\epsilon}(0) / \mu\right)+\left(\beta V_{2}(0) / \mu(\widehat{\lambda}-\right.$ $\eta))+(\epsilon / \mu \widehat{\lambda}) e^{\eta t}$, and $\beta(t)=(\beta \gamma / \mu(\widehat{\lambda}-\eta))=\rho$, one obtains

$$
u(t) \leq \alpha(t)+\int_{0}^{t} \beta(s) u(s) d s, \quad t \geq 0 .
$$

Then by using Lemma 5, it is easy to get

$$
u(t) \leq\left(\frac{V_{\epsilon}(0)}{\mu}+\frac{\beta V_{2}(0)}{\mu(\widehat{\lambda}-\eta)}\right) e^{\rho t}+\frac{\epsilon \eta}{\mu \widehat{\lambda}(\eta-\rho)} e^{\eta t}, \quad t \geq 0,
$$

which implies

$$
\begin{array}{r}
V_{\epsilon}(t) \leq\left(\frac{V_{\epsilon}(0)}{\mu}+\frac{\beta V_{2}(0)}{\mu(\hat{\lambda}-\eta)}\right) e^{-(\eta-\rho) t}+\frac{\epsilon \eta}{\mu \widehat{\lambda}(\eta-\rho)}, \\
t \geq 0 .
\end{array}
$$

Let $\epsilon \rightarrow 0^{+}$, one can get

$$
V_{1}(t) \leq e^{-(\eta-\rho) t}\left(\frac{V_{1}(0)}{\mu}+\frac{\beta V_{2}(0)}{\mu(\hat{\lambda}-\eta)}\right) .
$$


Then it follows from (31) that

$$
V_{2}(t) \leq e^{-\eta t} V_{2}(0)+e^{-(\eta-\rho) t}\left(\frac{V_{1}(0)}{\mu}+\frac{\beta V_{2}(0)}{\mu(\widehat{\lambda}-\eta)}\right) \frac{\gamma}{\rho} .
$$

Furthermore, it follows from condition (21) that $\eta>\rho$. Therefore together with (43) and (44), one can conclude that condition (17) is satisfied; that is, the entrained collective rhythms of multicellular systems (6) are achieved. The proof is thus completed.

Remark 8. The obtained result not only provides a new prospective to understand the interactions between the external stimuli and intrinsic physiological rhythms but also is potentially useful for the development of some medical devices. The result presented here is more effective in comparison with those in [38-41], where it is assumed that all the states are available for feedback purpose.

As a special case, if the positive matrices $P_{i}$ and $Q_{i}$ in the Lyapunov function (22) are chosen as the identity matrix, the following simplified result could be readily obtained.

Corollary 9. For a given scalar $\mu \in(0,1)$, if there exist scalars $\lambda_{i}>0, \mu_{i}>0, i=1,2, \ldots, l, \eta_{i}>0, i=l+1, \ldots, N$, and positive scalars $\epsilon_{1}, \epsilon_{2}, \delta_{1}$, and $\delta_{2}$ such that

$$
\begin{gathered}
-A^{T}-A+\epsilon_{1} I+\epsilon_{1}^{-1} L^{T} L+c \delta_{1} \sum_{j=1}^{N}\left|G_{i j}\right| \\
+c \delta_{1}^{-1} \sum_{j=1}^{l}\left|G_{j i}\right| \Gamma^{T} \Gamma \leq \lambda_{i}, \\
\left(I+E_{i k}\right)^{T}\left(I+E_{i k}\right) \leq \mu_{i},
\end{gathered}
$$

for $i=1, \ldots, l$ and

$$
\begin{gathered}
-A^{T}-A+\epsilon_{2} I+\epsilon_{2}^{-1} L^{T} L+c \delta_{2} \sum_{j=1}^{N}\left|G_{i j}\right| \\
+c \delta_{2}^{-1} \sum_{j=l+1}^{N}\left|G_{j i}\right| \Gamma^{T} \Gamma \leq-\eta_{i},
\end{gathered}
$$

for $i=l+1, \ldots, N$, and, for any impulsive time sequence $\left\{t_{k}\right\}$ satisfying

$$
\delta:=\sup _{k}\left\{t_{k+1}-t_{k}\right\}<\frac{\ln (1 / \mu)}{\eta+\lambda+(\beta \gamma) /(\eta \mu)},
$$

for $k=0,1,2, \ldots$, where $\mu=\max \left(\mu_{i}\right), \lambda=\max \left(\lambda_{i}\right), i=$ $1, \ldots, l$, and $\eta=\min \left(\eta_{i}\right), i=l+1, \ldots, N$, then the entrained collective rhythms of multicellular systems (6) are achieved.

\section{Numerical Example}

In this section, a synthetic multicellular system composed of $N$ Goodwin oscillators [33] is employed to illustrate the effectiveness of the proposed control strategy.

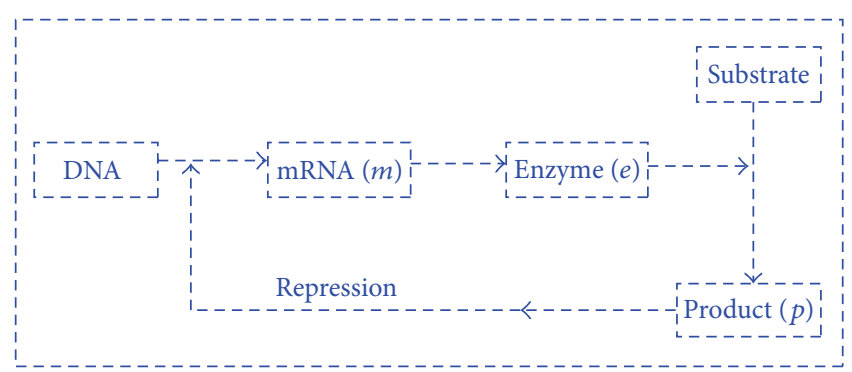

FIGURE 1: Regulatory scheme of the Goodwin oscillator.

4.1. Goodwin Oscillators. The Goodwin oscillator is a genetic network with negative feedback formed in a cyclic way [33], where metabolites repress the enzymes which are essential for their own synthesis by inhibiting the transcription of the molecule DNA to messenger RNA (mRNA) (see [42] for more details). The regulatory scheme of the Goodwin oscillator can be shown in Figure 1.

A modified model reflecting the above regulation process is given by

$$
\begin{aligned}
& \frac{d m}{d t}=\frac{u}{k_{1}+p^{\alpha}}-a m \\
& \frac{d e}{d t}=v m-b e \\
& \frac{d p}{d t}=w e-\frac{c p}{k_{2}+p}
\end{aligned}
$$

where $m, e$, and $p$ are the concentrations of mRNA, the enzyme, and the product of the reaction of the enzyme and a substrate, respectively. $a, b$, and $c$ are the degradation rates of each component, respectively. $u, v$, and $w$ denote the rates of transcription, translation, and catalysis, respectively. $k_{1}$ and $k_{2}$ are two positive constants. $\alpha$ is the Hill coefficient denoting the cooperativity of the end product repression. It should be pointed out that model (46) is slightly different from the model in [33]. By changing the linear degradation form of the product to Michaelis-Menten form, the limit cycle oscillations can occur for a lower value of the Hill coefficient $\alpha$ (see [42] for details).

The parameters are chosen as $a=b=c=v=w=0.1$, $u=1$, and $k_{1}=k_{2}=0.1$, and the Hill coefficient $\alpha$ is taken as $\alpha=4$. Given 4 random initial conditions, the simulation results are recorded in Figures 2 and 3. Figure 2 shows the time response of every products, and Figure 3 shows the limit cycle of the oscillators in phase space.

4.2. Multicellular System Model. Without loss of generality, we perform the simulation study on the scale-free network structure, which is assumed to obey the scale-free distribution of the Barabási-Albert (BA) model [43]. The parameters are given as $m_{0}=m=5$ and $N=60$. Figure 4 is the generated BA network graph.

The inner coupling matrix $\Gamma$ is given by $\Gamma=\operatorname{diag}(1,1.2,1)$, and the regulation function in Michaelis-Menten form satisfies condition (2). Assume that only the first 20 nodes are 


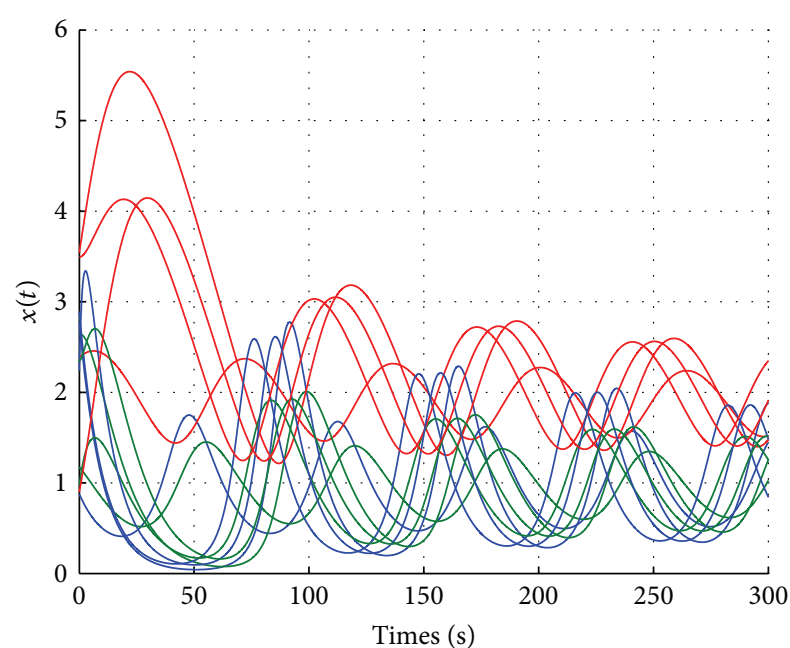

$x_{1}$
$-\quad x_{2}$
$-x_{3}$

FIGURE 2: Time response of four oscillators.

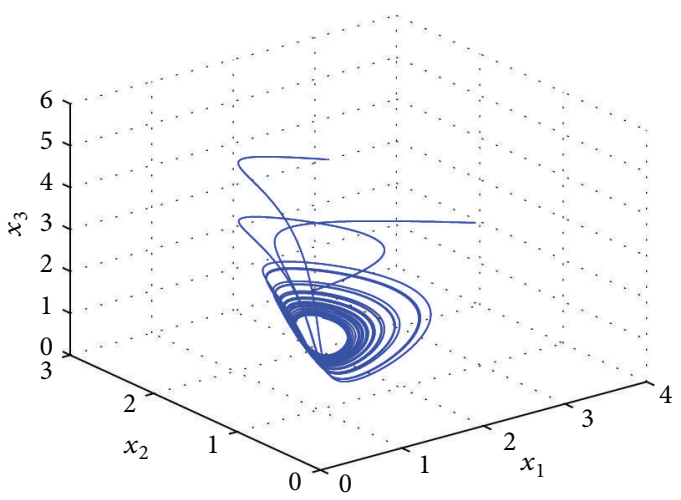

FIGURE 3: Limit cycle of the oscillators in phase space.

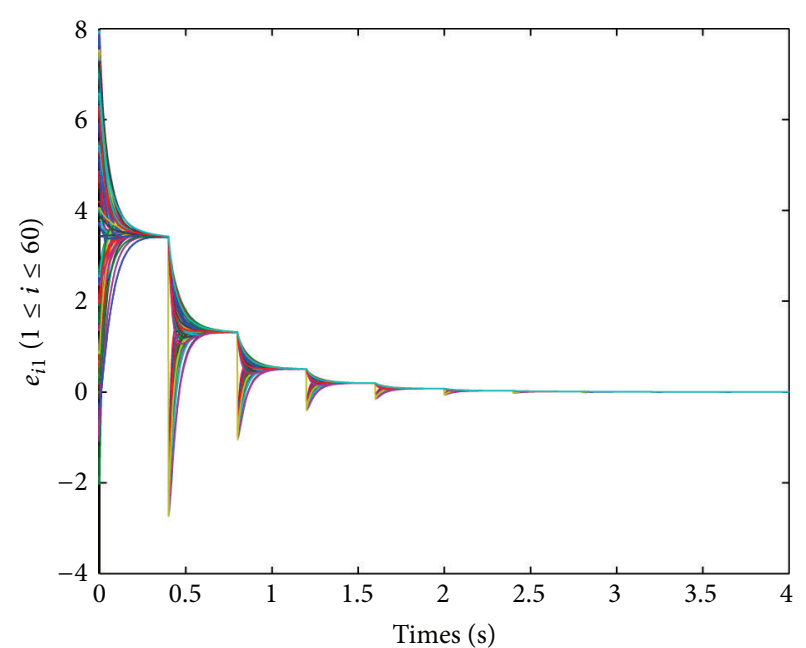

FIgURE 5: Rhythmic errors $e_{i 1}$ in BA network.

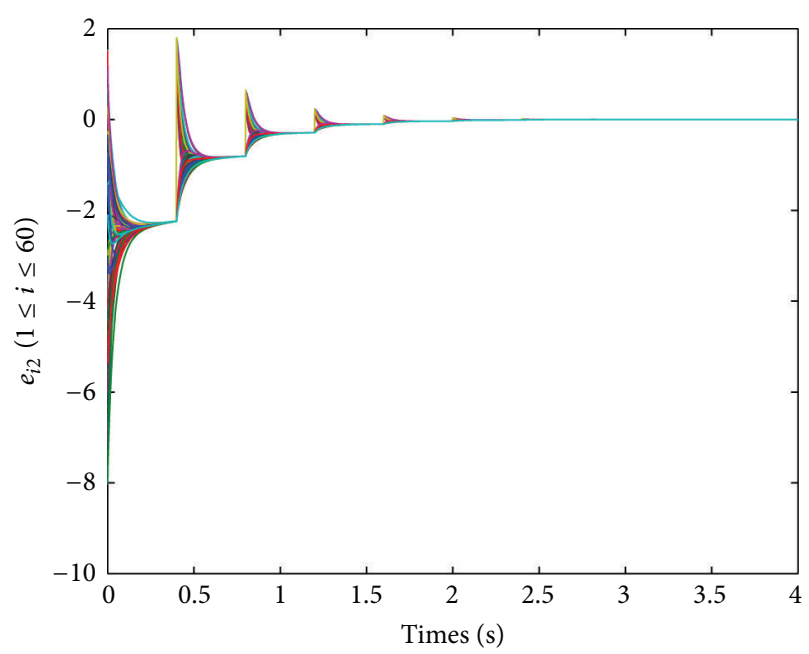

FIGURE 6: Rhythmic errors $e_{i 2}$ in BA network.

able to accept the external inputs. The parameters are chosen as $c=4, \epsilon_{1}=0.03$, and $\epsilon_{2}=0.01$. The impulse magnitude is given as $E_{i k}=-1.8$, and impulse interval is chosen as $\delta=0.4$. It follows from Corollary 9 that the entrained collective behaviors can be achieved. Figures 5, 6, and 7 show the rhythmic errors of the multicellular systems.

It is noted that since only 20 measurable cells in the network are subject to the external stimuli, the approaches in [38-41], which need the information of all the states, cannot be applied in this case.

\section{Conclusion}

In this paper, the entrained collective rhythms of multicellular systems have been investigated. It is shown that the entrained collective behavior can be achieved via impulsive control even when only partial states of multicellular systems are available. With the help of the newly developed impulsive integrodifferential inequality, the sufficient conditions are derived

FIGURE 4: BA scale-free network graph. 


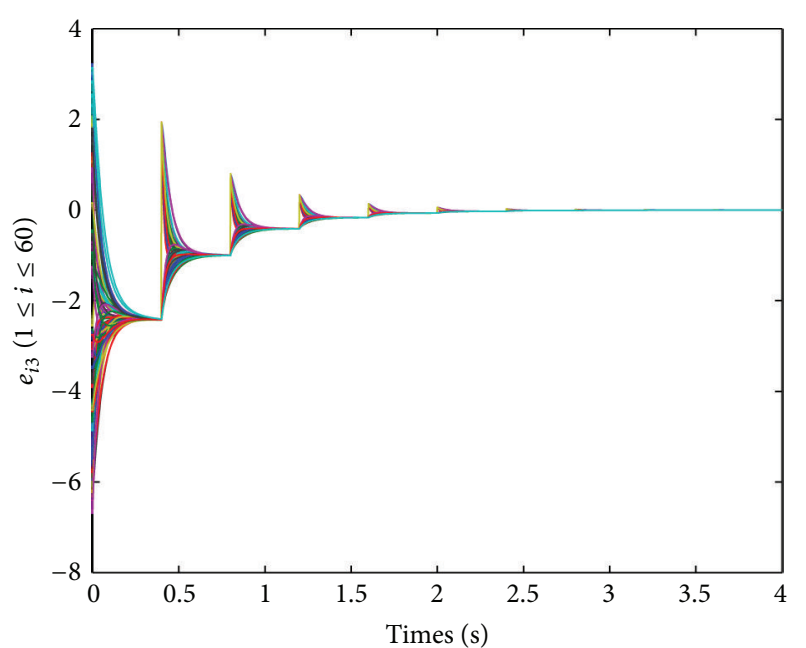

FIGURE 7: Rhythmic errors $e_{i 3}$ in BA network.

to ensure the entrained collective rhythms of multicellular systems. A synthetic multicellular system is finally used to illustrate the effectiveness of the developed impulsive control strategy.

\section{Acknowledgments}

The work was supported in part by the National Natural Science Foundation of China under Grant 61104045, in part by Technology Foundation for Selected Overseas Chinese Scholar, Ministry of Personnel of China, and in part by the Fundamental Research Funds for the Central Universities of China under Grant 2012B03514.

\section{References}

[1] L. Glass, "Synchronization and rhythmic processes in physiology," Nature, vol. 410, no. 6825, pp. 277-284, 2001.

[2] J. Garcia-Ojalvo, M. B. Elowitz, and S. H. Strogatz, "Modeling a synthetic multicellular clock: repressilators coupled by quorum sensing," Proceedings of the National Academy of Sciences of the United States of America, vol. 101, no. 30, pp. 10955-10960, 2004.

[3] D. McMillen, N. Kopell, J. Hasty, and J. J. Collins, "Synchronizing genetic relaxation oscillators by intercell signaling," Proceedings of the National Academy of Sciences of the United States of America, vol. 99, no. 2, pp. 679-684, 2002.

[4] T. Zhou, L. Chen, and R. Wang, "A mechanism of synchronization in interacting multi-cell genetic systems," Physica D, vol. 211, no. 1-2, pp. 107-127, 2005.

[5] E. Ullner, A. Koseska, J. Kurths, E. Volkov, H. Kantz, and J. García-Ojalvo, "Multistability of synthetic genetic networks with repressive cell-to-cell communication," Physical Review E, vol. 78, no. 3, Article ID 031904, 8 pages, 2008.

[6] T. Zhou, J. Zhang, Z. Yuan, and L. Chen, "Synchronization of genetic oscillators," Chaos, vol. 18, no. 3, Article ID 037126, 20 pages, 2008.

[7] Y. Sun, G. Feng, and J. Cao, "A new approach to dynamic fuzzy modeling of genetic regulatory networks," IEEE Transactions on Nanobioscience, vol. 9, no. 4, pp. 263-272, 2010.
[8] M. Mormont and F. Lévi, "Circadian-system alterations during cancer processes: a review," International Journal of Cancer, vol. 70, no. 2, pp. 241-247, 1997.

[9] J. Cao and F. Ren, "Exponential stability of discrete-time genetic regulatory networks with delays," IEEE Transactions on Neural Networks, vol. 19, no. 3, pp. 520-523, 2008.

[10] L. Pan and J. Cao, "Anti-periodic solution for delayed cellular neural networks with impulsive effects," Nonlinear Analysis: Real World Applications, vol. 12, no. 6, pp. 3014-3027, 2011.

[11] Y. Sun, G. Feng, and J. Cao, "Robust stochastic stability analysis of genetic regulatory networks with disturbance attenuation," Neurocomputing, vol. 79, pp. 39-49, 2012.

[12] Q. Zhu and J. Cao, "Stability analysis of Markovian jump stochastic BAM neural networks with impulse control and mixed time delays," IEEE Transactions on Neural Networks and Learning Systems, vol. 23, no. 3, pp. 467-479, 2012.

[13] S. Yamaguchi, H. Isejima, T. Matsuo et al., "Synchronization of cellular clocks in the suprachiasmatic nucleus," Science, vol. 302, no. 5649, pp. 1408-1412, 2003.

[14] R. Wang and L. Chen, "Synchronizing genetic oscillators by signaling molecules," Journal of Biological Rhythms, vol. 20, no. 3, pp. 257-269, 2005.

[15] C. Li, L. Chen, and K. Aihara, "Synchronization of coupled nonidentical genetic oscillators," Physical Biology, vol. 3, no. 1, pp. 37-44, 2006.

[16] C. Li, L. Chen, and K. Aihara, "Stochastic synchronization of genetic oscillator networks," BMC Systems Biology, vol. 1, article 6, 2007.

[17] J. Qiu and J. Cao, "Global synchronization of delay-coupled genetic oscillators," Neurocomputing, vol. 72, no. 16-18, pp. 3845-3850, 2009.

[18] P. M. Simon, A. M. Habel, J. A. Daubenspeck, and J. C. Leiter, "Vagal feedback in the entrainment of respiration to mechanical ventilation in sleeping humans," Journal of Applied Physiology, vol. 89 , no. 2, pp. 760-769, 2000.

[19] A. Wagemakers, J. Buldu, J. Garcia-Ojalvo, and M. Sanjuan, "Synchronization of electronic genetic networks," Chaos, vol. 16, no. 1, Article ID 013127, 8 pages, 2006.

[20] R. Wang, L. Chen, and K. Aihara, "Synchronizing a multicellular system by external input: an artificial control strategy," Bioinformatics, vol. 22, no. 14, pp. 1775-1781, 2006.

[21] T. F. Schultz and S. A. Kay, "Circadian clocks in daily and seasonal control of development," Science, vol. 301, no. 5631, pp. 326-328, 2003.

[22] Y. Li, Z. Liu, J. Zhang, R. Wang, and L. Chen, "Synchronisation mechanisms of circadian rhythms in the suprachiasmatic nucleus," IET Systems Biology, vol. 3, no. 2, pp. 100-112, 2009.

[23] J. Cao and L. Li, "Cluster synchronization in an array of hybrid coupled neural networks with delay," Neural Networks, vol. 22, no. 4, pp. 335-342, 2009.

[24] W. Yu et al., "Local synchronization of a complex network model," IEEE Transactions on Systems, Man, and Cybernetics B, vol. 39, no. 1, pp. 230-241, 2009.

[25] J. Lu, D. W. C. Ho, and J. Cao, "A unified synchronization criterion for impulsive dynamical networks," Automatica, vol. 46, no. 7, pp. 1215-1221, 2010.

[26] J. Lu, D. W. C. Ho, J. Cao, and J. Kurths, "Exponential synchronization of linearly coupled neural networks with impulsive disturbances," IEEE Transactions on Neural Networks, vol. 22, no. 2, pp. 329-335, 2011. 
[27] X. Yang, J. Cao, and J. Lu, "Stochastic synchronization of complex networks with nonidentical nodes via hybrid adaptive and impulsive control," IEEE Transactions on Circuits and Systems, vol. 59, no. 2, pp. 371-384, 2012.

[28] D. T. Kaplan, J. R. Clay, T. Manning, L. Glass, M. R. Guevara, and A. Shrier, "Subthreshold dynamics in periodically stimulated squid giant axons," Physical Review Letters, vol. 76, no. 21, pp. 4074-4077, 1996.

[29] T. Zhou, J. Zhang, Z. Yuan, and A. Xu, "External stimuli mediate collective rhythms: artificial control strategies," PLOS ONE, vol. 2, no. 2, article e231, 2007.

[30] G. Feng and J. Cao, "Master-slave synchronization of chaotic systems with a modified impulsive controller," Advances in Difference Equations, vol. 2013, article 24, 2013.

[31] X. Yang, J. Cao, and Z. Yang, "Synchronization of coupled reaction-diffusion neural networks with time-varying delays via pinning-impulsive controller," SIAM Journal on Control and Optimization, vol. 51, no. 5, pp. 3486-3510, 2013.

[32] J. Lu, D. W. C. Ho, J. Cao, and J. Kurths, "Single impulsive controller for globally exponential synchronization of dynamical networks," Nonlinear Analysis: Real World Applications, vol. 14, no. 1, pp. 581-593, 2013.

[33] B. C. Goodwin, "Oscillatory behavior in enzymatic control processes," Advances in Enzyme Regulation, vol. 3, pp. 425-438, 1965.

[34] T. S. Gardner, C. R. Cantor, and J. J. Collins, "Construction of a genetic toggle switch in Escherichia coli," Nature, vol. 403, no. 6767, pp. 339-342, 2000.

[35] M. B. Miller and B. L. Bassler, "Quorum sensing in bacteria," Annual Review of Microbiology, vol. 55, pp. 165-199, 2001.

[36] L. Huang, Linear Algebra in Systems and Control Theory, Science Press, Beijing, China, 1984.

[37] R. Bellman, "The stability of solutions of linear differential equations," Duke Mathematical Journal, vol. 10, pp. 643-647, 1943.

[38] W. Wang and J. Cao, "Synchronization in an array of linearly coupled networks with time-varying delay," Physica A, vol. 366, pp. 197-211, 2006.

[39] P. Li, J. Cao, and Z. Wang, "Robust impulsive synchronization of coupled delayed neural networks with uncertainties," Physica A, vol. 373, pp. 261-272, 2007.

[40] J. Cao, D. W. C. Ho, and Y. Yang, "Projective synchronization of a class of delayed chaotic systems via impulsive control," Physics Letters A, vol. 373, no. 35, pp. 3128-3133, 2009.

[41] Y. Yang and J. Cao, "Exponential synchronization of the complex dynamical networks with a coupling delay and impulsive effects," Nonlinear Analysis: Real World Applications, vol. 11, no. 3, pp. 1650-1659, 2010.

[42] C. P. Fall, E. S. Marland, J. M. Wagner, and J. J. Tyson, Eds., Computational Cell Biology, vol. 20 of Interdisciplinary Applied Mathematics, Springer, New York, NY, USA, 2005.

[43] A.-L. Barabási and R. Albert, "Emergence of scaling in random networks," Science, vol. 286, no. 5439, pp. 509-512, 1999. 


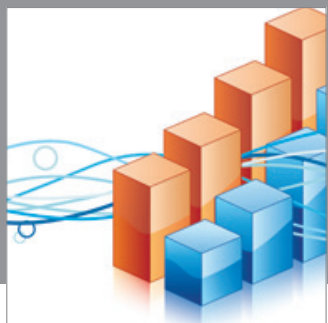

Advances in

Operations Research

mansans

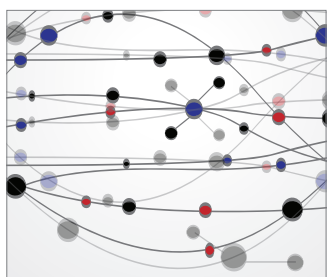

The Scientific World Journal
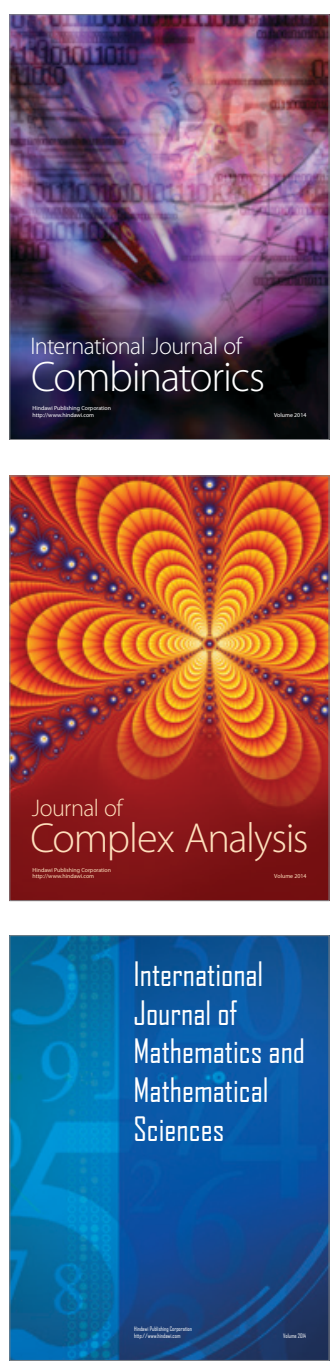
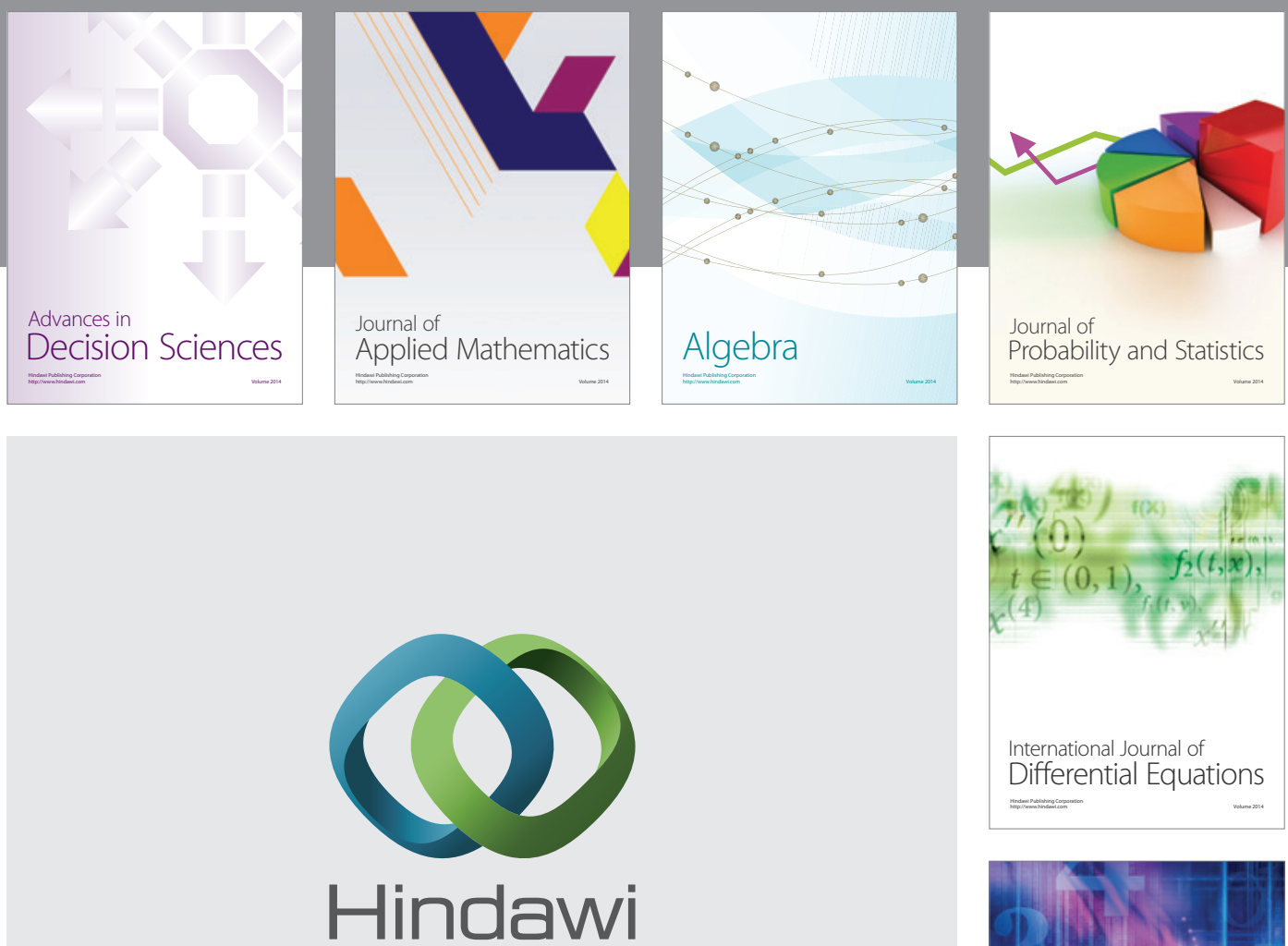

Submit your manuscripts at http://www.hindawi.com
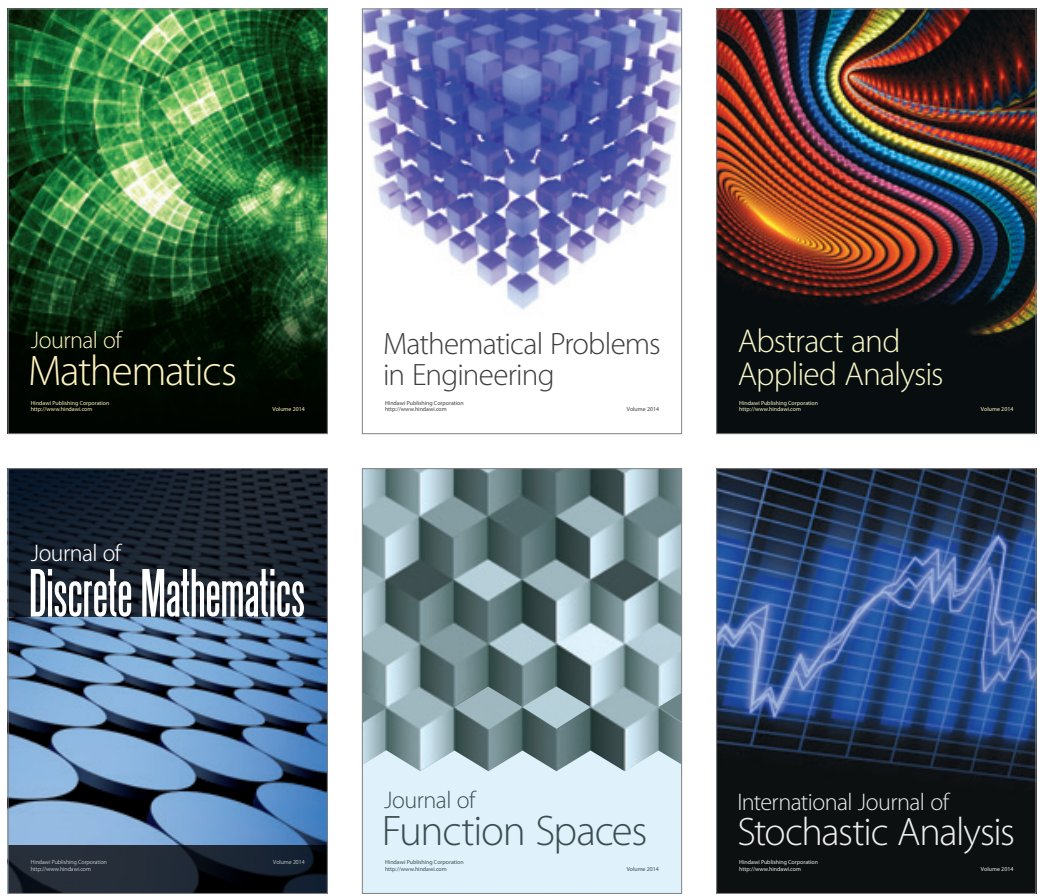

Journal of

Function Spaces

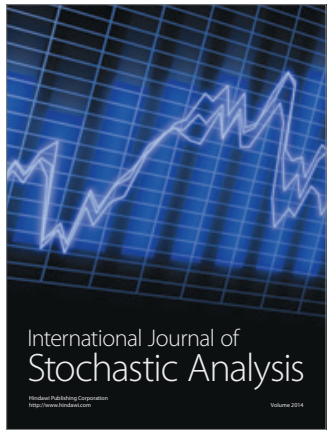

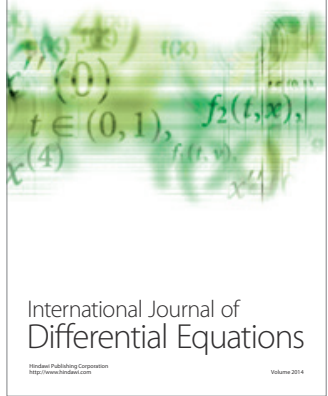
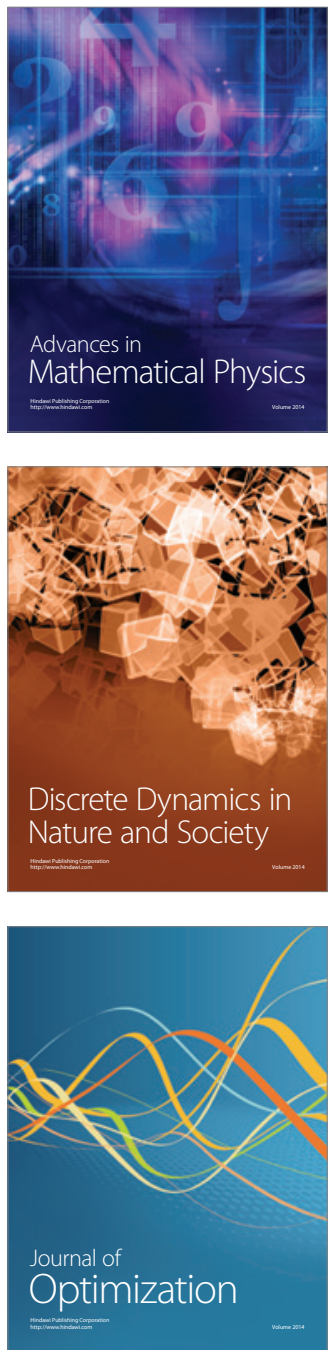